\title{
Franciscus Sanchez "Scepticus": un médecin philosophe précurseur de Descartes (1550-1623)
}

\author{
ELAINE LIMBRICK
}

François Sanchez, dit "Le Sceptique," médecin et philosophe toulousain, traduit dans son oeuvre philosophique cette grande incertitude que bien des penseurs de la Renaissance avaient éprouvée devant l'échec de la scolastique face aux nouvelles découvertes scientifiques. Son oeuvre se caractérise par une recherche ardente de la vérité, recherche d'une méthode qui permettrait un jugement sûr et complet sur le réel par la voie de la raison démonstrative. ${ }^{1}$ Adversaire acharné d'Aristote, comme de tout dogmatisme, Sanchez se rattache à l'école sceptique académique, allant jusqu'à signer dans une lettre adressée au célèbre mathématicien jésuite, Christophe Clavius, "Carneades philosophus," et se décrivant à la fin: "Sum enim alter Carneades, non gloriae inanis, sed veritatis, et tui amantissimus."2

On se demande pourquoi ce jeune médecin, en écrivant à un de ses anciens professeurs à Rome, se réclamait de Carnéade, l'un des dialecticiens les plus subtils de la Nouvelle Académie. On s'interroge sur les raisons qui l'ont poussé à écrire un traité sceptique des plus radicaux, intitulé le Quod nihil scitur, ouvrage qui a connu cinq éditions depuis sa publication en 1581 , et qui a suscité une âpre controverse en Allemagne au milieu du dix-septième siècle, pour tomber par la suite dans l'oubli le plus profond, semble-t-il, jusqu'à nos jours. ${ }^{3}$ Tâchons, dans la mesure du possible, d'apporter quelques réponses.

D'abord nous voudrions fournir quelques renseignements biographiques pour le situer mieux dans son cadre historique. Né à Tuy, dans la Galice espagnole, vers la seconde moitié de l'année 1550, François Sanchez était fils du docteur Antonio Sanchez, professeur de médecine réputé. ${ }^{4} \mathrm{Sa}$ famille se rattachait à la célèbre maison marrane, ou "Cristiana nueva," d'Aragon, qui occupa à la cour de Don Juan II (1458 à 1479) et à celle de Don Fernand V, Le Catholique (1479 à 1516), un rang très distingué jusqu'aux temps de la conspiration des Judéos conversós de Saragosse contre le Saint-Office (1484 à 1486). Après l'Edictogeneral de Expulsión du 31 mars, 1492, contre les Juifs, suivi de la Declaración du 18 mai, 1492, quelques familles israélites parmi lesquelles les Ram, certains Lópes, les Tarrega, passèrent en Guyenne, en Languedoc, et dans le midi de la France. D'autres Lópes, les Sanchez, 
cherchèrent asile, parait-il, d'abord aux confins des Galices espagnole et portugaise, et ensuite, vers le milieu de seizième siècle, décidèrent d'émigrer en France. ${ }^{5} \mathrm{Ce}$ fut le cas de la famille de Sanchez.

Après ses premières études à Tuy, François Sanchez fut envoyé dans la vieille capitale de la Galice, à l'école célèbre de Braga, pour y suivre ses cours d'enseignement secondaire. ${ }^{6}$ Après l'installation de son père à Bordeaux, le jeune Sanchez continua ses études supérieures, jusqu'à la maîtrise ès arts, au Collège de Guyenne pendant les années 1565 à 1571, où il commença ses études de médecine. ${ }^{7}$ Puis il entreprit un voyage en Italie, visitant les universités du nord (Pise, Bologne, Padoue, Venise, Ferrare et Florence) avant de s'installer chez un membre de sa famille, Duarte Paulo, à Rome où il acheva sa première formation médicale à la Faculté de la Sapienza, y prenant connaissance des découvertes anatomiques de Vésale, de Fallope, et de Columbo. ${ }^{8}$ Là, il fit la connaissance du célèbre géomètre jésuite, Christophe Clavius, à qui il envoya ses premiers écrits, ses Objectiones et Erotemata super geometricas Euclidis Demonstrationes, dont le manuscrit a malheureusement disparu.

Sanchez s'étant fait immatriculer à la faculté de médecine de Montpellier dès octobre 1573, fut promu bachelier en médecine le 23 novembre 1573, sous le parrainage du professeur François Feynes, reçut sa licence en avril, et son doctorat en juillet, 1574. Carrière brillante, rapide! Tout de suite il présenta sa candidature à la chaire de médecine que le décès de François Feynes avait laissée vacante mais la chaire fut donnée à un huguenot, Antoine Saporta, fils de l'ancien professeur et chancelier Jean Saporta. Déçu dans ses ambitions, et contraint par les guerres de religion à quitter cette ville huguenote, Sanchez se réfugia à Toulouse, ville catholique, où l'université était surtout célèbre pour ses théologiens, ses juristes, et ses philosophes. ${ }^{9}$ L'arrivée de Sanchez à Toulouse fin février, début mars 1575, annonce une période de recueillement philosophique très intense avant qu'il n'assume ses fonctions de médecin en 1581 .

L'inscription sous le portrait de Sanchez qu'on trouve encore dans la galerie de la Faculté de médecine de Toulouse nous donne des renseignements précieux sur la carrière de cet homme remarquable. On y lit:

Le Tudéen, Don Francisco Sánchez, dit le Sceptique (1550-1623), Professeur Royal de Philosophie et de Médecine à Toulouse, Recteur de l'université de cette ville. Doyen de la Faculté des Arts et plus tard de la Faculté de Médecine.

Quen s'est-il donc passé pour qu'un jeune médecin se métamorphose en philosophe sceptique, pour qu'il soit entré par postulation à la Faculté des Arts de l'université vers 1580 , et qu'il y ait donné des cours de philosophie? Or, les titres de Sanchez lui permettaient d'enseigner la philosophie tout en attendant d'être nommé médecin et professeur dans la Faculté de Médecine. Les cours 
de médecine, d'ailleurs, consistaient en des commentaires très savants des textes d'Hippocrate et de Galien. Un nouvel arrêt (25 mars 1571) ordonnait aux deux professeurs de la Faculté de Médecine: "oultre les leçons publicques qu'ils sont tenus continuer au temps ordonné par les statuts, faire deux anathomies tous les ans pour le moins et tous aultres exercices de leurs Facultés." ${ }^{10}$ Comme Sanchez avait été formé par un disciple de Vésale à Rome, il lui fut facile de donner des cours d'anatomie.

Consultons son premier cours de philosophie, donné à la Faculté des Arts, et qui constitue le préambule à son traité, le De Divinatione Per Somnum ad Aristotelem, paru à Lyon en 1576. Ce traité, dirigé contre Jérôme Cardan, nous donne une idée de son itinéraire philosophique traversé entre les années 1575 et 1581 , date de la publication du Quod nihil scitur, bien que ce traité-ci fût déjà rédigé dès $1574 .^{11}$

Depuis longtemps nous avions résolu de nous taire et de nous livrer en silence et pour nous mêmes à la philosophie, plutôt que de déraisonner ouvertement avec tant d'insensés, d'avérer à tous notre folie par ses disputes, ses leçons publiques comme aussi par ses ouvrages: et qui pis est, peut-être, si du moins, il en advient ainsi, d'entraîner autrui dans la même folie.

Qu'est-ce autre chose notre savoir, en effet, qu'une confiance téméraire jointe à une ignorance absolue? Ou qui ose prétendre connaître à fond quelque chose de ce que renferme le sein immense de la nature, au milieu enfin, d'une obscurité et de ténèbres si profondes? alors que maîtres et disciples s'accordent si peu? alors que notre esprit est si misérable et si faible? alors que tant de difficultés le cernent et l'écrasent de toute part? ${ }^{12}$

Cependant, poussé par la considération de son devoir et de sa charge, Sanchez décida alors d'être "fou avec les autres ou, si nous sommes seuls à déraisonner, nous mesurer à la folie des autres."13 Tout comme Montaigne dans sa tour, ou Descartes dans son "poêle," Sanchez se livra à de longues méditations sur le problème de la connaissance: "Je lisais les préceptes des anciens, je sondais les pensées de mes contemporains." ${ }^{14}$ Mais nulle part il ne trouve de réponse satisfaisante, car l'aristotélisme dominait encore toute la pensée scientifique et religieuse. Sanchez se lança avec toute la fougue de sa jeunesse dans la lutte contre le dogmatisme. Se modelant sur Carnéade, ce dialecticien remarquable et défenseur de la doctrine probabiliste, Sanchez n'hésita pas à accabler cette prétendue science des Aristotéliciens avec tout l'arsenal des arguments sceptiques. Pour lui la connaissance demeure relative car le critère de la vérité est purement subjectif. La règle suffisante pour la vie pratique sera la probabilité. Or le probabilisme, tel que Cicéron le présente dans le Lucullus, est une doctrine qui convient au sage et qui est la plus favorable aux progrès dans les sciences: "Sic quidquid acciderit specie probabile, si nihil se offeret quod sit probabilitati illi contrarium, utetur eo sapiens, ac sic omnis ratio vitae gubernabitur" (Academica II, 31). Ainsi la pensée philosophique de Sanchez, comme nous allons voir, renforce et confirme sa pensée scientifique. 
Les écrits philosophiques de Sanchez qui nous sont parvenus ne sont pas nombreux. Au début de 1578 il fit paraître le Carmen de Cometa anni 1577, composé contre le Discours sur ce que menace devoir advenir la comete apparue à Lyon de 12 de ce mois de Novembre de 1577 de François Junctini. Il fit publier ensuite à Lyon en 1581 son Quod nihil scitur sur lequel repose principalement sa renommée. Ses autres écrits philosophiques ne furent publiés qu'après sa mort, à Toulouse, dans ses Opera medica de 1636, grâce aux soins de ses deux fils et de son élève, Raymond Delassus. Ces traités philosophiques sont le De longitudine et brevitate vitae liber; le In librum Aristotelis Physiognomicon commentarius, et le De Divinatione per somnum ad Aristotelem. D'autres écrits philosophiques furent projetés, tel le De modo sciendi, dont Sanchez fait mention à la fin du Quod nihil scitur mais les manuscrits furent perdus ou ne virent jamais le jour.

Oeuvre philosophique dont la minceur surprend et qui est attribuable, peut-être, aux exigences de son double emploi comme professeur à la Faculté des Arts et médecin à l'Hôtel-Dieu de Toulouse (il y fut nommé en janvier 1582 et resta pendant trente ans au service de l'hôpital charitable). Nous savons que Sanchez devint professeur à la Faculté des Arts au début de l'année 1585, la chaire de philosophie lui ayant été attribuée par postulation. ${ }^{15}$ Comme Joaquim Serrão nous l'indique, Sanchez resta "vingt-sept ans titulaire de sa chaire de Philosophie et y acquit un immense prestige." $16 \mathrm{Ce}$ fut seulement après la mort de son compatriote, Manuel Alveres, que Sanchez entra dans la Faculté de Médecine, en 1612, comme titulaire de la seconde chaire d'Hygiène et Thérapeutique. ${ }^{17}$ L'énorme corpus de ses oeuvres médicales témoigne de son intérêt pour les sciences pragmatiques qui correspondrait à ses préoccupations philosophiques. Galéniste enthousiaste, Sanchez n'hésitera pas, cependant, à critiquer son maître au nom de l'expérience. ${ }^{18}$ Ainsi, dans son commentaire sur le De Pulsibus de Galien, Sanchez avertit ses étudiants: "Cum vero tota controversia sit de facto non de iure, rationibus, non pugnandum esset, sed experimento" (Opera medica, 1636, p. 589).

En effet, les fondements de tout son enseignement médical reposent sur un système de pensée sceptique où toute hypothèse est rejetée si elle ne s'accorde point avec l'observation et l'expérience. Rappelons toujours que Sanchez travaille dans les limites des données scientifiques de son époque. Mais il brûle du désir d'unir la médecine à la philosophie et en parle en ces termes dans son traité, De Longitudine et Brevitate Vitae liber: "Ultimo denique, ut Medicinam Philosophiae conjungamus, qua maxime ratione vita hominis produci possit, generalibus quibusdam praeceptis docebimus" (in Opera medica, p. 14).

Le Quod nihil scitur reste, cependant, l'oeuvre essentielle pour comprendre la pensée sanchésienne. La controverse qui s'était élevée en Allemagne après la parution de la cinquième édition à Stettin en 1665, sous le titre paradoxal, Sanchez aliquid sciens, éclaire l'importance de ce petit traité dans la restauration du scepticisme moderne, importance que Bayle avait reconnue 
dans son Dictionnaire Historique et Critique. Le savant allemand, Daniel Hartnack, fit accompagner le texte du Quod nihil scitur d'amples notes et commentaires qui servaient à réfuter totalement les thèses de Sanchez, utilisant les arguments contre le scepticisme empruntés à Aristote, à Cicéron et à saint Augustin, dont la célèbre formule - "Qui dubitat: an aliquid scire contingat; se dubitare non dubitat" - donnera lieu aux arguments avancés par Descartes dans le Discours de la Méthode et les Méditations métaphysiques.

Tout comme Montaigne au début de "l'Apologie de Raimond Sebond," et plus particulièrement au début de l'essai "De l'experience," Sanchez s'attaque à la notion même de "science," commentant ainsi la première phrase de la Métaphysique d'Aristote:

Le désir de savoir est inné à l'homme. [Mais, dit Sanchez,] "Tous les hommes veulent savoir; quelques-uns seulement savent vouloir; un plus petit nombre encore parviennent à savoir. Pour moi, je n'ai pas eu plus d'heur que les autres. Dès mon enfance, je me suis appliqué à l'étude de la nature et je m'informais minutieusement de toutes choses. Au début, mon esprit avide de savoir se contentait de toute nourriture qu'on lui présentait, quelle qu'elle fût; mais au bout de peu de temps, il fut pris d'indigestion et se mit à tout rejeter. Je cherchai alors ce que je pourrais bien lui offrir qu'il fût capable de comprendre parfaitement et dont il pût tirer pleinement profit; mais personne ne pouvait combler mon souhait. Je retournais les dires des anciens; je sondais les pensées des contemporains; ils me répondaient de même; mais je n'y trouvais rien absolument qui pût me satisfaire. Quelques-uns, je l'avoue, me montraient une ombre de vérité; mais je n'en trouvai aucun qui pût me déclarer franchement et sans réserves quel jugement on devait porter sur les choses. Je rentrai donc en moi-même, et révoquant toutes les opinions en doute, comme si aucune n'eût jamais été exprimée par personne, je me mis à examiner les choses en elles-mêmes; ce qui est la vraie méthode du savoir."19

Histoire d'une déception intellectuelle que Descartes racontera pareillement au début de son Discours de la Méthode:

J'ai été nourri aux lettres dès mon enfance, et, pource qu'on me persuadait que, par leur moyen, on pouvait acquérir une connaissance claire et assurée de tout ce qui est utile à la vie, j'avais un extrême désir de les apprendre. Mais, sitôt que j'eus achevé tout ce cours d'études, au bout duquel on a coutume d'être reçu au rang des doctes, je changeai entièrement d'opinion. Car je me trouvais embarrassé de tant de doutes et d'erreurs, qu'il me semblait n'avoir fait autre profit, en tâchant de m'instruire, sinon que j'avais découvert de plus en plus mon ignorance. $^{20}$

Descartes part en voyage par la suite, dans le but de trouver "dans le grand livre du monde," la réponse à ses doutes, mais il trouvera, en considérant les moeurs d'autres hommes "quasi autant de diversité qu'il avait fait auparavant entre les opinions des philosophes" (p. 10). Ce fut alors qu'il prit la résolution, tout comme Sanchez, "de ne chercher plus d'autre science, que celle qui se 
pourrait trouver en moi-même" (p. 9). Chez Descartes le doute universel, voire hyperbolique, est une étape nécessaire de la conscience passant de l'erreur à la certitude subjective.

Il faut bien comprendre que ce qui anime les réflexions philosophiques d'un Sanchez et d'un Descartes, c'est le désir de trouver une véritable méthode sur laquelle fonder des bases indiscutables de la science. ${ }^{21}$ Devant l'échec de l'aristotélisme, il devint nécessaire d'avoir recours à un autre système de pensée pour expliquer la nature des choses. Or, pour Descartes les vérités mathématiques offraient un moyen de s'évader du scepticisme et de construire une science certaine. Mais cette voie ne convenait pas à Sanchez qui, dans une lettre envoyée à Clavius, en parle dans ces termes:

\begin{abstract}
J'avais parcouru le champ de la Physique et de la Métaphysique, et n'y avais pas trouvé la vérité. Comme je continuais à m'informer à son sujet, certains me répondirent qu'elle avait établi sa demeure dans une région intermédiaire entre la nature et le monde transcendant, c'est-à-dire dans le domaine des mathématiques. J'accours donc à ce domaine avec joie et curiosité. Mais un général éprouvé, lorsqu'il voit grandes ouvertes les portes d'une ville ennemie, ne s'y introduit pas témérairement; il soupçonne des embûches, fait tout examiner par des éclaireurs, et sonde tous les recoins; ou, quand il envahit une province ennemie, il ne laisse derrière lui aucun ouvrage fortifié sans le détruire et le raser; de même, en abordant le terrain des mathématiques, je me suis mis à hésiter, à redouter de tous les côtés la ruse; je m'entourai de soupçons et de défiance. Bien m'en a pris; sans cela je serais tombé dans une multitude de pièges, de fosses dont est creusé le terrain des mathématiques; elles ne sont pas si nombreuses et si vastes que dans la physique ou la métaphysique; mais elles sont par là-même plus difficiles à éviter et plus redoutables. ${ }^{22}$
\end{abstract}

Selon Sanchez, les mathématiques ne peuvent pas fournir le modèle de la science, car leurs démonstrations ne sont point indubitables, ni exemptes d'erreur. Tout compte fait, les théorèmes mathématiques sont établis sur des propositions hypothétiques et non pas sur des données empiriques.

Dans son Quod nihil scitur Sanchez avance beaucoup plus loin que Montaigne, par exemple, sur la voie de l'empirisme sceptique, ce qui n'est pas étonnant si l'on considère les paroles de Sextus Empiricus sur la différence entre la médecine empirique et le scepticisme. ${ }^{23}$ Il veut prouver que la proposition dogmatique SCIENTIA EST REI PERFECTA COGNITIO, qui se réclame des méthodes scolastiques de la définition et de la démonstration, ne peut être réalisée. Aussi Sanchez combattra-t-il ces docteurs qui construisent une science purement verbale, les condamnant sévèrement pour leurs subtilités dialectiques:

En quel labyrinthe de mots nous entraînent-ils, loin de tout fondement de vérité! On ne saurait ainsi parvenir à la connaissance de la nature, à l'intelligence des choses réelles; ce sont des choses nouvelles et fictives dont ils nous expliquent la structure; mais il est impossible à l'esprit d'en obtenir l'intelligence. Comment avoir l'intelligence de ce qui n'est pas? ${ }^{24}$ 
Et Sanchez de citer comme exemples de ces objets d'une science verbale "les atomes de Démocrite, les Idées de Platon, les Nombres de Pythagore, les Universaux d'Aristote, l'Intellect agent ... ." 25 Car, pour lui, ce sont "des attrape-nigauds par où ces auteurs font croire qu'ils ont découvert des choses inconnues et les secrets de la Nature."26

Pour Sanchez, sceptique académique, la connaissance humaine est établie sur les données des sens, qui ne révèlent que les qualités extérieures, ou au mieux, les accidents. Néanmoins le sujet connaissant peut espérer avoir une connaissance imparfaite des choses par les moyens de l'expérience et du jugement ("experimentum iudiciumque"). "Aucun des deux," nous dit Sanchez à la fin de son traité, "ne peut se soutenir sans l'autre: quel usage et quel emploi on doit faire de chacun d'eux, c'est ce que nous exposerons plus amplement dans un prochain livre qui est en préparation. En attendant, il est visible que nous ne savons rien." 27 C'est donc grâce à la méthode empirique que l'homme peut atteindre une certaine perception des choses. Le Quod nihil scitur se termine, paradoxalement, par la promesse de fonder une science assurée et l'annonce d'un traité de méthode qui, malheureusement, ne nous est point parvenu.

Ainsi le scepticisme académique de Sanchez, tout comme le scepticisme interrogatif de Montaigne, et le doute hyperbolique de Descartes, est positif à la longue: le doute est un point de départ, une démarche préliminaire de l'esprit pour saisir le réel, avant d'élaborer une methode. Le "Quid?" par lequel Sanchez terminait ses traités médicaux et philosophiques fait écho au "Que sais-je?" de Montaigne et laisse le champ libre aux spéculations de la raison discursive. De même le doute universel chez Descartes est une machine à détecter l'évidence, une négation méthodique pour trouver la vérité. ${ }^{28}$

Pour conclure, en proclamant que l'homme ne peut rien savoir, Sanchez affirme que la science, comme connaissance absolue et parfaite, n'existe qu'en Dieu. Déjà, dans sa lettre à Clavius, il avait écrit:

Je passe sur un premier point: c'est que les études mathématiques méritent à peine le nom de sciences; car les sciences font appel aux sens plutôt qu'à l'esprit; c'est de là qu'elles tirent leur certitude, s'il est ici bas quelque chose de certain. La science véritable, en effet, c'est de connaître Dieu d'abord, puis la nature qui lui est soumise: la connaître du dedans et du dehors, comme on dit, ou comme dit Aristote, connaître une chose par ses causes. ${ }^{29}$

A maintes reprises dans le Quod nihil scitur et dans ses autres traités philosophiques, Sanchez souligne la nescience de l'homme comparée à la science de Dieu. Echos de Nicolas de Cuse, disent certains critiques, parmi eux Emilien Senchet.$^{30}$ Nous croyons plutôt, d'après nos propres recherches, que Sanchez suit la doctrine de la Nouvelle Académie, comme il l'indique à la fin de son traité médical, In librum Galeni de pulsibus ad tyrones commentarii: "Quae tamen iudicanda aliis relinquimus, et Academicorum more nihil 
affirmantes, cuique liberam eligendi potestatem concedimus" (p. 606). Or, le scepticisme académique, par son accent sur le phénomène, sur la subjectivité de la représentation, ne nie pas l'existence de l'objet en soi, et admet une transcendance. Ainsi, dans son Quod nihil scitur, avec des accents qui rappellent l'apologétique pascalienne, Sanchez évoquera la disproportion entre l'homme et Dieu:

Quam si perfectam [cognitionem] haberet, Deo similis esset, imo Deus ipse. Nec enim perfecte cognoscere potest quis, quae non creavit ... Imperfectus autem et miser homunculus quomodo cognoscet alia, qui seipsum non nosse potest, qui in se est, et secum? (p. 30)

En effet, il existe au delà du monde phénoménal une réalité transcendante qui, pour le sceptique chrétien, est Dieu. Reprenant à son compte le célèbre texte de l'Exode dans lequel Moïse demande à Yahweh son nom, et où Dieu répond "Je suis celui qui suis," Sanchez dira dans son traité De longitudine et brevitate vitae, où il examine la pensée d'Aristote sur la génération et sur la mort: "Ego sum qui sum. Esse Dei non tempore mensuratur, sed totum praesens totum simul est." 31 Dieu, pour Sanchez, est infini, intemporel, immensurable, et surtout incompréhensible. Il faut donc renoncer à cette belle raison humaine, trop incertaine, trop contradictoire, pour parvenir à la vraie connaissance qui repose en Dieu. Sanchez dit "Le Sceptique" ne manquera presque jamais de terminer ses traités médicaux et philosophiques par la prière "Laus Deo Virginique Mariae,". et sa piété, sa dévotion chrétienne sont attestées par son disciple Raymond Delassus. Le sceptique chrétien reconnaît les limites de sa raison, et s'incline, humblement, devant le mystère d'un Dieu caché.

\section{Université de Victoria}

\section{Notes}

1 Voir Francisco Sanches, Quod Nihil Scitur in Opera Philosophica, Nova edição, precedida de Introdução por Joaquim de Carvalho (Coimbra: 1955), p. 2. Sauf avis contraire, toutes nos références renvoient à ce recueil, qui est le plus accessible au lecteur moderne.

2 Sanches, Opera Philosophica, pp. 146, 153.

3 Voir Franciscus Sanchez, Quod nihil scitur (Lyon: 1581), (Francfort: 1618), in Opera medica (Toulouse: 1636), De multum nobili et prima universali scientia quod nihil scitur (Rotterdam: 1649), in Daniel Hartnack, Sanchez Aliquid Sciens (Stettin: 1665).

4 Voir la vie de Sanchez par Raymond Delassus dans la préface aux Opera medica (Toulouse: 1636), ẽ recto. Grâce aux recherches d'Henri-Pierre Cazac, dont les papiers sont conservés à l'Institut Catholique de Toulouse, nous avons des détails biographiques précis.

5 Voir le Fonds Cazac, Boîte II. Nous ne partageons pas les denières hypothèses émises par Richard H. Popkin dans la nouvelle édition de son The History of Scepticism from Erasmus to Spinoza (Berkeley and Los Angeles: University of California Press, 1979) p. 263, n. 48. Que Montaigne soit cousin éloigné de Sanchez, sa mère, Antoinette de Louppes [Lopez], étant descendante de juifs portugais, 


\section{2 / Renaissance and Reformation}

natifs de Valença do Minho, où la tante de Sanchez avait épousé un riche bourgeois du nom d'Antonio Lopez, nous voulons bien le croire. Cependant, il n'y a aucune preuve que les deux philosophes se soient rencontrés ni qu'ils aient lu leurs oeuvres respectives. On peut parler, certes, de sources communes de leur scepticisme; Diogène Laërce, Plutarque, Cicéron, Galien, et saint Augustin. Leur scepticisme fidéiste ne cache pas un cryptojudaïsme.

6 Voir Jean de Launoy, De scholis celebrioribus in Opera Omnia, IV (Paris: 1672).

7 Voir Ernest Gaullieur, Histoire du Collège de Guyenne (Paris: Sandoz et Fischbacker, 1874), pp. 29-30.

8 Voir Salvatore Miccolis, Francesco Sanchez (Bari: Tipografia Levante, 1965), pp. 13-15.

9 Voir Pierre Bayle, Dictionnaire historique et critique, $3^{\mathrm{e}}$ édit. (Rotterdam: 1720), article SANCHEZ, François.

10 Voir Joaquim Verissimo Serrão, Les Portugais à l'Université de Toulouse (Paris: 1970), p. 131.

11 "Cum nuper librorum scrinium evolverem, amicissime Jacobe, incidi forte in opusculum hoc, quod ante septennium edideram" Quod nihil scitur, p. 1.

12 Traduction de Cazac, Fonds Cazac, Boîte III.

13 Ibid.

14 "Evolvebam praeteritorum dicta, tentabam praesentium corda," Quod nihil scitur, p. 2.

15 Serrão, Les Portugais à l'Université de Toulouse, p. 146.

16 Ibid., p. 147.

17 Ibid., p. 150.

18 Voir Sanchez, Opera medica (Toulouse: apud Petrum Bosc., 1636), p. 611.

19 Cité d'après Joseph Moreau. Aucune traduction française du Quod nihil sciturn'a été publiée jusqu'ici. Voir son article magistral, "Doute et Savoir chez Francisco Sanches," Portugiesische Forschungen des Görresgesellschaft, Erste Reihe, Aufsätze zur Portugiesischen Kulturgeschichte, I, Band, (1960), pp. 26-27. Nous sommes très reconnaissants au professeur Moreau de tous ses conseils judicieux.

20 Descartes, Discours de la Méthode, ed. Etienne Gilson, $4^{\mathrm{e}}$ éd. (1925: Paris: J. Vrin, 1967) p. 4.

21 "Nihil enim tantum in docendo momentum habet, quantum methodus: quae subinde tam varia hominibus est: quaque uti scire non minus laboriosum, ingenioque plenum est, quam utile; nec minus rarum, quam necessarium," Sanchez, Quod nihil scitur, p. 44.

22 Cité d'après Moreau, pp. 31-32.

23 Voir Sextus Empiricus, Oeuvres Choisies de Sextus Empiricus, trad. Jean Grenier et Geneviève Goron (Paris: Aubier, 1948), pp. 210-211.

24 Cité d'après Moreau, p. 35.

25 Ibid., p. 35.

26 Ibid., p. 35.

27 Ibid., pp. 41-42.

28 Voir Henri Gouhier, "Doute méthodique ou négation méthodique?" Etudes Philosophiques 9, (1954), 135-162.

29 Cité d'après Moreau, p. 32.

30 Voir Emilien Senchet, Essai sur la Méthode de Francisco Sanchez (Paris: V. Giard et E. Brière, 1904), p. 6.

31 Sanchez, De Longitudine et Brevitate Vitae Liber, p. 7 in Opera medica (Toulouse: apud Petrum Bosc, 1636). Cf. Quod nihil scitur, p. 25 où Sanchez parle de Dieu dans les mêmes termes: "nostrorum Deus, cuius nulla mensura, nulla finitio nec subinde a mente comprehensio aliqua esse potest." 